\title{
An Algorithmic Analog-to-Digital Converter Using Unity-Gain Buffers
}

\author{
SATOMI OGAWA, STUDENT MEMBER, IEEE, AND KENZO WATANABE, SENIOR MEMBER, IEEE
}

\begin{abstract}
An algorithmic stage for bipolar 1-b analog-to-digital (A/D) conversion using a unity-gain buffer is proposed. Cyclic and pipeline A/D converter architectures using this stage iteratively or in cascade are also described. Error analysis and SPICE simulations show that a conversion accuracy higher than 8-b and a conversion rate up to $10 \mathrm{Mb} / \mathrm{s}$ are attainable with presently available 3- $\mu \mathrm{m}$ CMOS technologies. Video frequency operation may also be possible with finer linewidths. The component requirement is minimum, and thus it is best suited for an analog interface in ASIC. A prototype converter built using discrete components has confirmed the principles of operation.
\end{abstract}

\section{INTRODUCTION}

W ITH THE advance in digital signal processing and VLSI technologies, a system-on-chip is increasingly becoming prevalent. This trend is not limited to fully digital systems but extends to mixed digital and analog systems. A key component in such an analog ASIC is an analog-to-digital (A/D) converter for interfacing the real analog world with the digital world of signal processing. Typically such interfaces are successive approximation and oversampling delta-sigma A/D converters [1], [2]. These converters permit high accuracy A/D conversion, but their conversion speed is inadequate for video signal processing.

For digital processing of video or higher frequency signals, flash or parallel A/D converters, now available in CMOS monolithic form, are used exclusively. Their resolution is, however, limited to $8 \mathrm{~b}$, and their accomodation into an ASIC chip seems difficult, because of the extravagant hardware. Another promising candidate for high-speed high-accuracy A/D conversion is a pipeline method [3]. A pipeline converter can be realized by connecting 1-b algorithmic A/D stages in cascade, and thus requires only $n$ comparators for $n$-bit resolution. Many architectures using switched-capacitor techniques have already been proposed for such a stage, but almost all of them suffer from the charge injection due to clock feedthrough in analog switches [4]-[6]. In addition, op-amps used for the algorithmic operation limit the conversion rate to $5 \mathrm{Mb} / \mathrm{s}$ or less [4], [5].

An algorithmic stage using a unity-gain buffer is developed to alleviate these difficulties. The device count is the least of all pipeline architectures proposed so far. Follow-

Manuscript received February 14, 1990; revised June 22, 1990.

The authors are with the Research Institute of Electronics, Shizuoka University, Hamamatsu, 432 Japan.

IEEE Log Number 9038792. ing this introduction, principles of operation, cyclic and pipeline A/D converter architectures, conversion accuracy, and speed expected from their CMOS monolithic implementations will be described. A prototype cyclic A/D converter breadboarded using discrete components will be also presented to confirm the principles of operation.

\section{Architectures}

A recursive algorithm for an $n$-bit bipolar A/D conversion is expressed as follows [7]:

$$
\begin{aligned}
& V(i+1)=2 V(i)+(-1){ }^{b_{i}} V_{r} \\
& b_{i}= \begin{cases}1, & \text { if } V(i) \geqq 0 \\
0, & \text { otherwise }\end{cases} \\
& (i=1,2, \cdots, n)
\end{aligned}
$$

where $V_{r}$ is the reference voltage, $V(i)$ is the analog voltage sequence starting with $V(1)=V_{a}, V_{a}$ is an analog voltage ranging from $-V_{r}$ to $V_{r}$, and $b_{i}$ is the offset binary representation of $V_{a}$.

A new architecture for executing the recursive algorithm (1) is shown in Fig. 1 . Here, $C_{1}$ and $C_{2}$ are matched unit capacitors, and $\phi$ and $\bar{\phi}$ are the nonoverlapping two phase clocks. If $b_{i}$, the value determined in the preceding stage, is 1 , then $C_{1}$ and $C_{2}$ are charged to $V(i)$ and $V(i)$ $-V_{r}$, respectively, in the $\phi=" 1$ " phase. In the next $\bar{\phi}$ $=$ " 1 " phase, the charge stored in $C_{2}$ is fully discharged into $C_{1}$. The output voltage $V(i+1)$ of a unity-gain buffer is then $2 V(i)-V_{r}$. If $b_{i}=0$, on the other hand, then $C_{1}$ and $C_{2}$ are both charged to $V(i)$ in the $\phi=$ " 1 ", phase. In the next $\bar{\phi}=$ "1" phase, the charge stored in $C_{2}$ is fully discharged into $C_{1}$ and the reference voltage source is connected in series with $C_{1}$. The ouput voltage of the unity-gain buffer thus becomes $2 V(i)+V_{r}$. Therefore, this stage executes the recursive algorithm (1) in one clock cycle. Detecting the polarity of the output voltage $V(i+1)$ in the $\bar{\phi}=$ " 1 " phase, the comparator (CP) determines the value of $b_{i+1}$.

In this architecture, if the switching sequence is arranged such that $M_{2}$ or $M_{3}$ is turned off prior to $M_{1}$, then the feedthrough charge from $M_{2}$ or $M_{3}$ charges only $C_{2}$, while that from $M_{1}$ charges only $C_{1}$. If switches $M_{1}, M_{2}$, and $M_{3}$ consist of matched MOS transistors, $C_{1}$ and $C_{2}$ store the same amount of feedthrough charge but in opposite polarity. These charges cancel each other in the subsequent charge transfer in the $\bar{\phi}=$ " 1 " phase, and 


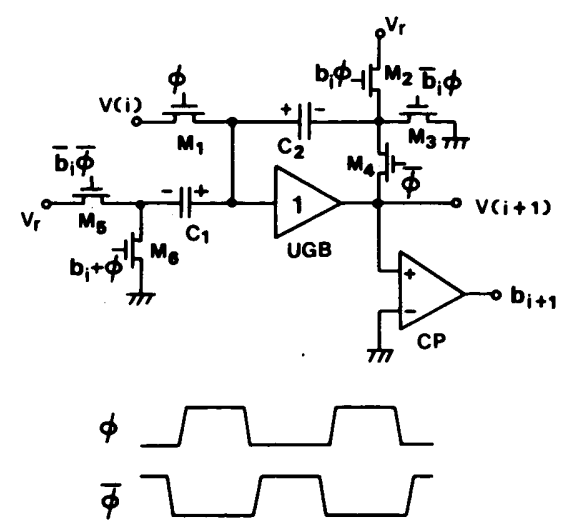

Fig. 1. A new architecture of the 1-b quantizer.

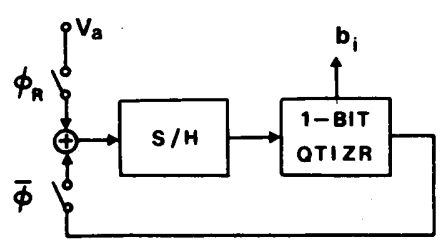

(a)

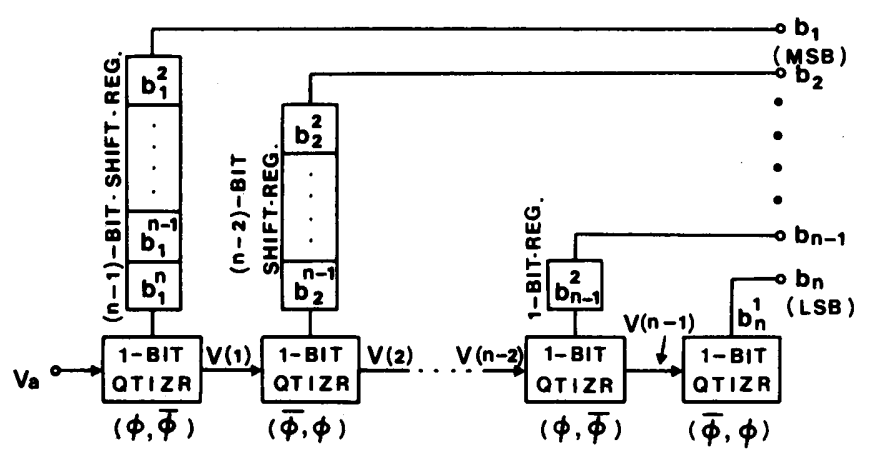

(b)

Fig. 2. (a) Block diagrams of cyclic and (b) pipeline A/D converters

thus no offset voltage due to clock feedthrough appears at the output. The offset voltage of the unity-gain buffer can also be canceled by slightly modifying the circuit configuration of the unity-gain buffer and switching sequence [8]. Therefore, this stage allows an accurate 1-b A/D conversion.

Cyclic and pipeline A/D converters can be realized using this stage iteratively or in cascade, as shown in Figs. 2(a) and (b). In Fig. 2(a), the sample/hold (S/H) circuit samples the output voltage of the unity-gain buffer in the $\bar{\phi}=$ " 1 " phase and holds it during the next $\phi=$ " 1 ", phase to update the input of the unity-gain buffer. This process is repeated $n$ times, and thus $n$ clock cycles are required for an $n$-bit $A / D$ conversion. In the pipeline architecture of Fig. 2(b), the odd stages perform the sample operation in the $\phi=$ " 1 " phase, and determine the values of $b_{i}$ in the $\bar{\phi}=$ " 1 " " phase, while the even stages sample the outputs of the preceding stages in the $\bar{\phi}=$ " 1 " phase and execute the algorithm (1) in the $\phi=$ " 1 " phase, to determine the value of $b_{i}$. Therefore, accomplishing the $n$-bit conversion in $n / 2$ clock cycles, the pipeline A/D converter updates the digital output in every clock cycle, and thus high-speed A/D conversion can be expected.

\section{Conversion Accuracy}

The main error sources involved in the present $A / D$ architecture are the gain error of the unity-gain buffer, the offset voltages of the unity-gain buffer, the charge injection due to clock feedthrough, and capacitance mismatch between $C_{1}$ and $C_{2}$. In the following, the effect of each error source is estimated separately.

Let the gain of the unity-gain buffer be $1-\delta$. The recursive algorithm executed by the 1-b quantizer is then modified to

$$
V^{\prime}(i+1)=\frac{1-\delta}{1+\delta}\left\{2 V^{\prime}(i)+(-1)^{b_{i}} V_{r}\right\}
$$

where a prime denotes the erroneous quantity. Executing (3), the 1-b quantizer produces the integral nonlinearity which depends on the bit pattern. Fig. 3 shows the simulation result of the integral nonlinearity when $\delta$ is $0.2 \%$, showing that the error voltage becomes maximum when all bits assume 1 or 0 . If this error is smaller than the voltage corresponding to a $1 \mathrm{LSB}$, then no missing code appears and the conversion is accurate down to its LSB. Formulating this 1 LSB accuracy condition, we have, to first order:

$$
\delta\left\{2^{n+1}(1-n \delta)-n+1\right\}<1 .
$$

The gain error $\delta$ can be reduced to $0.1 \%$ or less by the improved buffer configuration described in [9]. Thus a conversion accuracy higher than $9 \mathrm{~b}$ can be obtained.

If the offset voltage $V_{\text {os }}$ is present in the 1-b quantizer, then the algorithm is modified to

$$
V^{\prime}(i+1)=2 V^{\prime}(i)+(-1)^{b_{i}} V_{r}+2 V_{\text {os }} .
$$

The error voltage due to the offset voltage is accumulated independent of the bit pattern and amounts, after $n$ interations, to $2^{n} V_{\text {os }}$. Fig. 4 shows the differential nonlinearity due to the offset voltage, indicating that it becomes maximum at the major carry. If these errors are less than 1 LSB, then no missing code appears. Formulating this condition, we have

$$
\left|V_{\mathrm{os}}\right|<\frac{V_{r}}{2\left(2^{n}-1\right)}
$$

The offset voltage of a CMOS buffer is typically a few millivolts. Therefore, clock feedthrough dominates the offset voltage of the 1-b quantizer. If the clock feedthrough and offset cancellation scheme described in Section II is used, the offset voltage can be reduced to $1 \mathrm{mV}$ of less. Then, a conversion accuracy limited by the offset voltage is estimated to be $10 \mathrm{~b}$ when $V_{r}=2 \mathrm{~V}$.

Assume now that capacitance mismatch between $C_{1}$ and $C_{2}$ is $\epsilon$. Then the algorithm is modified to

$$
V^{\prime}(i+1)=(2-\epsilon) V^{\prime}(i)+(-1+\epsilon)^{b_{i}} V_{r} .
$$

The integral nonlinearity caused by $\epsilon$ becomes maximum at the major carry, as shown in Fig. 5. For the maximum 


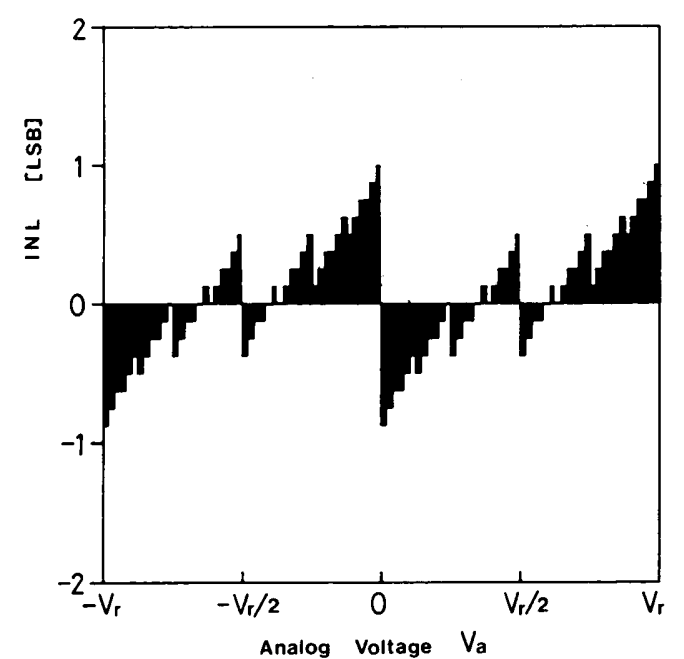

Fig. 3. The integral nonlinearity in $8-\mathrm{b} A / D$ conversion when the gain error of the unity-gain buffer is $0.2 \%$.

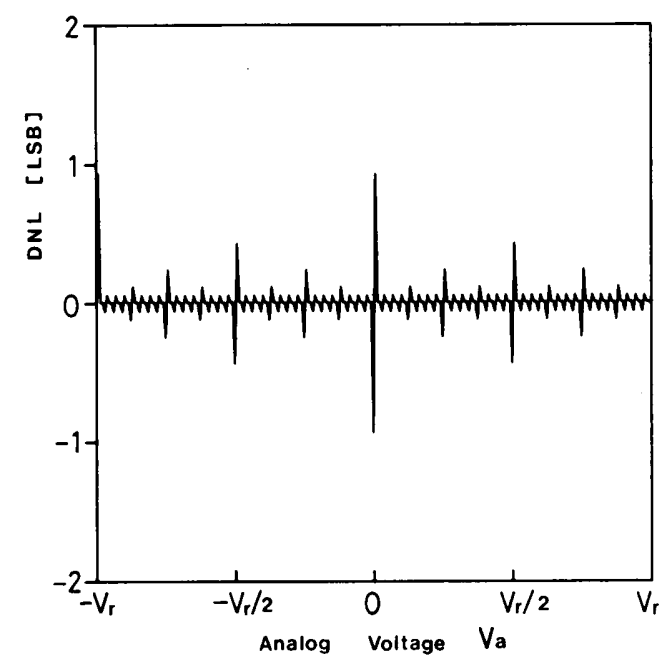

Fig. 4. The differential nonlinearity in 8 -b $A / D$ conversion when the offset voltage of the $1-b$ quantizer is $7 \mathrm{mV}$.

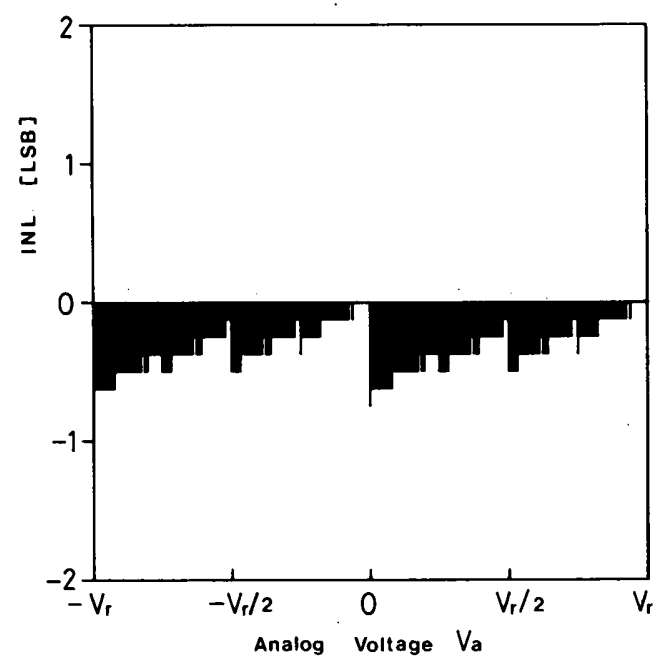

Fig. 5. The integral nonlinearity in $8-\mathrm{b} A / D$ conversion when the capacitance mismatch is $0.2 \%$.

error to be less than $1 \mathrm{LSB}, \epsilon$ should satisfy, to first order, the condition

$$
\epsilon(2-\epsilon)^{n-1}<1 .
$$

Capacitance mismatch $\epsilon$ less than $0.4 \%$ is attainable with presently available CMOS technologies. Therefore, a conversion accuracy higher than $9 \mathrm{~b}$ can be obtained.

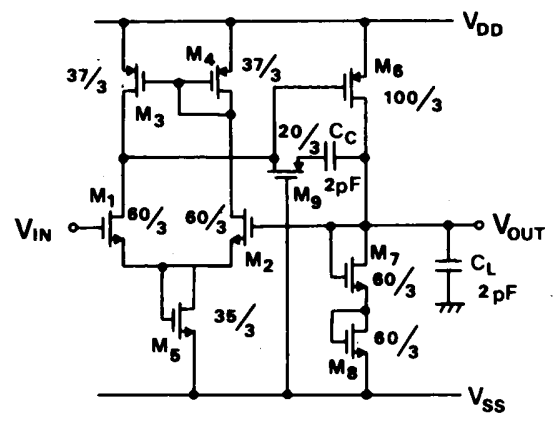

Fig. 6. Circuit diagram of an op-amp-based unity-gain buffer.

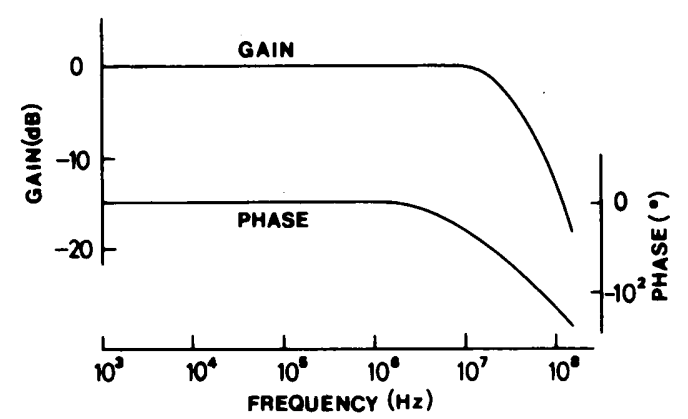

Fig. 7. The frequency response of the op-amp-based unity-gain buffer.

Summarizing the above discussions, one can conclude that a conversion accuracy higher than $8 \mathrm{~b}$ can be obtained by CMOS monolithic realization of the present A/D architectures.

\section{Conversion Speed And Power Consumption}

As described in Section II, the present architectures consist basically of unity-gain buffers, comparators, and digital circuitry. A key component in CMOS realization is unity-gain buffer, because comparators and digital circuitry have already been well established. In this section, a CMOS unity-gain buffer is described to estimate the conversion speed and power consumption.

Fig. 6 shows the circuit diagram of a CMOS op-amp based unity-gain buffer. The gain is very close to 1 , and the error is less than $0.1 \%$ even if the input pair $\left(M_{1}, M_{2}\right)$ and the current mirror $\left(M_{3}, M_{4}\right)$ are subjected to the threshold variation encountered in a normal process. The frequency response is shown in Fig. 7 . It can be seen that pole/zero cancellation by $C_{c}$ and $M_{9}$ compensates completely the phase characteristics [10] and the unity-gain frequency extends to $10 \mathrm{MHz}$.

A conversion rate is limited by the $0.1 \%$ settling time $t_{s}$ of unity-gain buffer, the propagation delay $t_{d}$ of the digital circuitry, and the response time $t_{r}$ of the comparator. Fig. 8 shows the transient response of the unity-gain buffer. The output voltage of the unity-gain buffer settles within $40 \mathrm{~ns}$ even under large signal operation. $t_{d}$ and $t_{r}$ of the 3- $\mu \mathrm{m}$ CMOS digital circuitry and comparator are estimated by SPICE simulations to be 30 and $20 \mathrm{~ns}$, respectively. Therefore, a conversion rate up to $10 \mathrm{Mb} / \mathrm{s}$ can be expected from monolithic realization using a $3-\mu \mathrm{m}$ process, and a higher rate for finer linewidths.

Circuit parameters of the unity-gain buffer are chosen such that a slew rate is $250 \mathrm{~V} / \mu \mathrm{s}$ when $V_{\mathrm{DD}}=-V_{\mathrm{SS}}=$ $5 \mathrm{~V}$ and a load capacitor $C_{L}$ is $2 \mathrm{pF}$. The power dissipated 


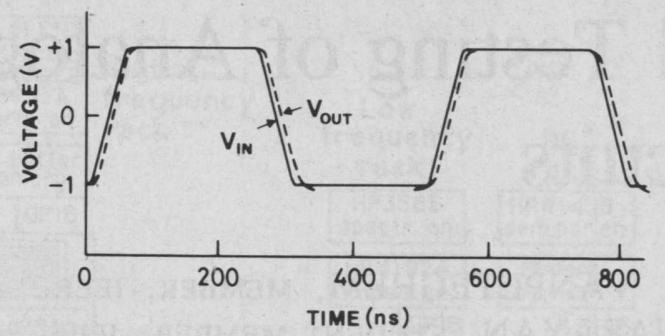

Fig. 8. The transient response of the op-amp-based unity-gain buffer.

in the unity-gain buffer is then $5 \mathrm{~mW}$. Thus a total power consumption of the 8-b pipeline A/D converter, including the power dissipated in comparators and digital circuitry, is estimated to be $50 \mathrm{~mW}$, which is ten times smaller than that of an 8-b CMOS flash converter.

\section{A Prototype Cyclic A/D Converter}

To confirm the principles of operation, an 8-b cyclic A/D converter based on Fig. 2(a) was built using discrete components. The unity-gain buffer and the $\mathrm{S} / \mathrm{H}$ circuit were formed using LF347 op-amps. The switches used were MC14016 and capacitors were all $4.7 \mathrm{nF}$. Capacitance mismatch between $C_{1}$ and $C_{2}$ was less than $0.5 \%$.

Fig. 9 shows experimentally observed waveforms when the prototype cyclic A/D converter converted the analog voltage $V_{a}=-0.71 \mathrm{~V}$ into a binary number with a reference voltage $V_{r}=2 \mathrm{~V}$. In the first cycle of the conversion state, the polarity of $V_{a}$ is detected. In this case, $V_{a}$ $<0$, and thus $b_{1}$ is set to 0 (bottom trace). In the next cycle, the analog voltage sequence (lower middle trace) $V(2)=2 V_{a}+V_{r}=0.58 \mathrm{~V}$ is produced by the $1-\mathrm{b}$ quantizer and its polarity is detected by a comparator again. Since $V(2)>0, b_{2}$ is set to 1 . Repeating this process 8 times, the converter produces $b=(01010010)_{B}$, which is the correct digital equivalent of the analog input voltage.

Fig. 10 shows the transfer characteristics of the prototype converter. Missing codes can be seen appearing at the major carries. These errors are attributed to the offset voltage and clock feedthrough in the quantizer, and hence, can be reduced greatly by using the previously mentioned cancellation techniques.

\section{CONCLUSIONS}

An algorithmic stage using a unity-gain buffer was proposed for 1-b quantization, and cyclic and pipeline A/D converters using this stage iteratively or in cascade were described. Error analysis and SPICE simulations show that a conversion accuracy higher than $8 \mathrm{~b}$ and a conversion rate up to $10 \mathrm{Mb} / \mathrm{s}$ can be expected from their monolithic realization using a $3-\mu \mathrm{m}$ CMOS process. A higher rate for video signal processing is also possible with finer linewidths. The component requirement is minimum, and thus the architectures described herein can be integrated onto a small chip area. The power consumption is also very small. Therefore, it is best suited for an analog interface in ASIC. Its CMOS implementation and dynamic tests to evaluate the noise floor and effective bits are future works.

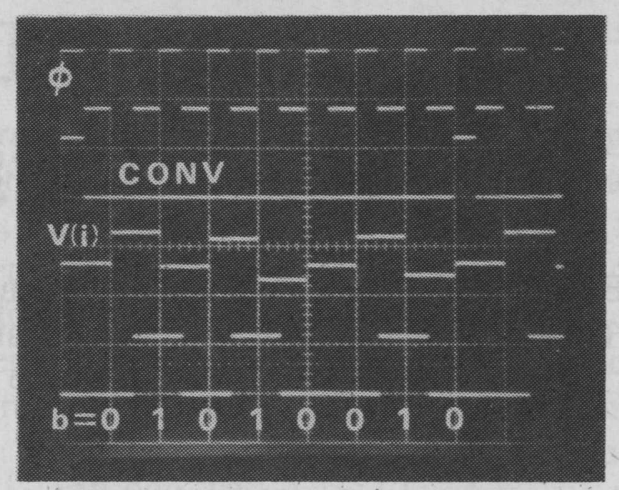

Fig. 9. Experimentally observed clock signal (top trace), reset signal (upper middle trace), analog voltage sequence $V(i)$ (lower middle trace), and comparator output $b$ (bottom trace) when the prototype cyclic A/D converter converted the analog voltage $-0.71 \mathrm{~V}$ into an 8 -b binary number with a voltage reference $V_{r}=2 \mathrm{~V}$. Horizontal scale: $50 \mu \mathrm{s} /$ div; vertical scale: $10 \mathrm{~V} /$ div (top, upper middle and bottom traces) and 2 $\mathrm{V} /$ div (lower middle trace).

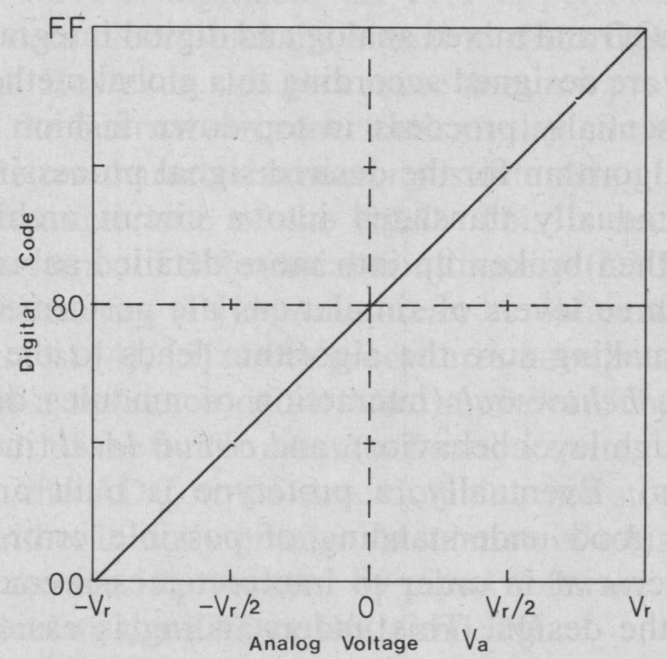

Fig. 10. The transfer characteristics of the prototype 8-b cyclic converter.

\section{REFERENCES}

[1] J. L. McCreary and P. R. Gray, "All-MOS charge redistribution analog-to-digital conversion techniques-Part I," IEEE J. Solid-State Circuits, vol. SC-10, pp. 371-379, Dec. 1975.

[2] R. Koch, B. Heise, F. Eckbauer, E. Engelhardt, J. A. Fisher, and F. Parzefall, "A 12-bit sigma-delta analog-to-digital converter with a 15-MHz clock rate,' IEEE J. Solid-State Circuits, vol. SC-21, pp. 1003-1010, Dec. 1986.

[3] G. C. Temes, F.-J. Wang, and K. Watanabe, "Novel pipeline data converters,' in Proc. IEEE Int. Symp. Circuits Syst., pp. 1943-1946, 1988.

[4] S. Masuda, Y. Kitamura, S. Ohya, and M. Kikuchi, "A CMOS pipeline algorithmic A/D converter," in Proc. Custom Integrated Circuits Conf., pp. 559-562, 1984.

[5] K. Martin, "A high speed, high accuracy pipeline A/D converter,' in Rec. Asilomar Conf. Circuits, Syst. and Comput., pp. 489-492, 1981.

[6] S. H. Lewis and P. R. Gray, “A pipelined 5-M sample/s 9-bit analogto-digital converter," IEEE J. Solid-State Circuits, vol. SC-22, pp. 954-961, Dec. 1987

[7] H. Schmit, Electronic Analog/Digital Conversions. New York: Reinhold, 1970, p. 195.

[8] K. Watanabe and S. Ogawa, "Clock-feedthrough compensated sample/hold circuits,"' Electron. Lett., vol. 24, pp. 1226-1228, Sept. 1988.

[9] G. C. Temes, "High accuracy pipeline A/D converter configuration," Electron. Lett., vol. 21, pp. 762-763, Aug. 1985.

[10] R. Gregorian and G. C. Temes, MOS Analog Integrated Circuits for Signal Processing. New York: Wiley, 1986, ch. 4. 